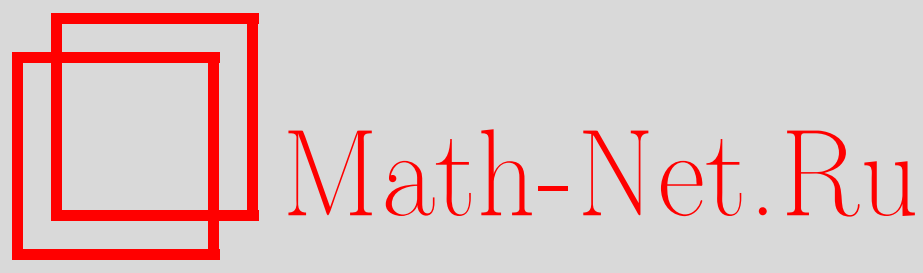

Р. Фалкао, А. Франсиско Нето, Э. Перейра, Аналитический подход к (ан)гармоническим кристаллическим цепочкам с самосогласованными стохастическими резервуарами, ТМФ, 2008, том 156, номер 1, 138-146

DOI: https://doi.org/10.4213/tmf6235

Использование Общероссийского математического портала Math-Net.Ru подразумевает, что вы прочитали и согласны с пользовательским соглашением http: //www.mathnet.ru/rus/agreement

Параметры загрузки:

IP : 3.85 .5 .30

26 апреля 2023 г., $17: 37: 27$

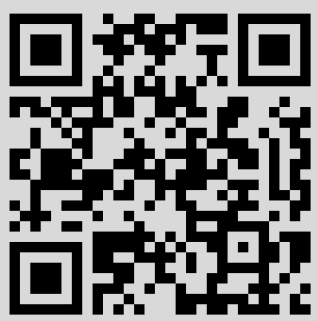




\title{
АНАЛИТИЧЕСКИЙ ПОДХОД \\ К (АН)ГАРМОНИЧЕСКИМ КРИСТАЛЛИЧЕСКИМ ЦЕПОЧКАМ С САМОСОГЛАСОВАННЫМИ СТОХАСТИЧЕСКИМИ РЕЗЕРВУАРАМИ
}

\begin{abstract}
Рассмотрены гармонические и ангармонические цепочки осцилляторов с самосогласоваными стохастическими резервуарами и выведено интегральное представление (в духе Фейнмана-Каца) для корреляций, в частности для потока тепла. Для гармонической цепочки дается новое доказательство конечности ее теплопроводности в стационарном состоянии. Подход, опирающийся на это интегральное представление для корреляций и на пертурбативный анализ, имеет весьма общий характер и может быть распространен на более сложные системы.
\end{abstract}

Ключевые слова: (ан)гармонические кристаллы, стохастические резервуары, поток тепла.

\section{1. ВВЕДЕНИЕ}

В неравновесной статистической механике аналитический вывод макроскопических термодинамических законов для микроскопических моделей взаимодействующих частиц представляет собой вызов теоретикам. В частности, даже в одномерном контексте нет строгого вывода закона теплопроводности Фурье, согласно которому поток тепла пропорционален градиенту температуры [1]. Многие работы, почти все с использованием компьютерных вычислений [2] и с результатами, противоречащими другу другу, были посвящены этой теме со времени первых строгих исследований по гармоническим цепочкам осцилляторов с термостатами на границах [3] модели, которая не подчиняется закону Фурье. Недавно к гармонической цепочке осцилляторов вернулись в рамках аналитических исследований, но при условии, что каждый узел взаимодействует со стохастическим резервуаром. Было доказано [4],

${ }^{*}$ Centro de Formação de Professores, UFRB Amargosa, BA, Brazil.

E-mail: rfalcao@fisica.ufmg.br

${ }^{\dagger}$ Núcleo de Física, UFS Itabaiana, Brazil. E-mail: afneto@fisica.ufs.br

${ }^{\ddagger}$ Departamento de Física-ICEx, UFMG, Belo Horizonte, Brazil.

E-mail: emmanuel@fisica.ufmg.br 
что в этом случае имеет место закон Фурье. Разумеется, если нет взаимодействия с внутренними резервуарами, теплопроводность расходится, и закон Фурье более не выполняется, как ранее было показано в [3]. Поскольку методика, предложенная в работе [4], целиком зависела от линейности динамики (т.е. от гармоничности потенциала), некоторые из авторов настоящей работы предложили другой подход к исследованию цепочки осцилляторов с резервуаром в каждом узле с целью изучения задачи с ангармоническими потенциалами и выяснения их поведения при наличии термостатов только на границах [5]-[8]. Предложенный подход опирается на интегральное представление для корреляций (связанных с тепловым потоком), которое анализируется пертурбативным методом.

В настоящей статье, продолжая наши предыдущие теоретические работы (включающие, как было сказано, ангармонические взаимодействия), мы строго выводим интегральное представление для корреляций (и тем самым для потока тепла). Более того, мы обращаемся к более простому случаю гармонической цепочки с резервуаром в каждом узле и даем новое доказательство конечности теплопроводности, используя наш подход и пертурбативный анализ (мы доказываем сходимость ряда теории возмущений). Представленное здесь выражение для теплопроводности совпадает с выражением из [4].

Опишем нашу модель. Рассмотрим решеточную систему с неограниченными скалярными переменными в пространстве $\Lambda \subset \mathbb{Z}$ и со стохастическим термостатом в каждом узле. Точнее, мы возьмем систему $N$ осцилляторов с гамильтонианом

$$
H(p, q)=\sum_{j=1}^{N} \frac{1}{2}\left(p_{j}^{2}+M q_{j}^{2}\right)+\frac{1}{2} \sum_{\substack{j, l=1 \\ j \neq l}}^{N} q_{j} J_{j l} q_{l}+\sum_{j=1}^{N} \lambda \mathcal{P}\left(q_{j}\right),
$$

где $J_{l j}=J_{j l}, J_{l j}=f(|l-j|), \mathcal{P}\left(q_{j}\right)$ - ангармонический потенциал, например $\mathcal{P}\left(q_{j}\right)=q_{j}^{4}$ ( $\varphi^{4}$-модель жесткой ангармоничности) или $\mathcal{P}\left(q_{j}\right)=1-\cos \left(q_{j}\right)$ (модель Френкеля-Конторовой мягкой ангармоничности). Как правило, индексы $j$ и $l$ выбираются соседними, причем $J_{j, j+1}=J$ для любого $j$. В этом случае межчастичные взаимодействия можно также записать в виде

$$
\sum_{j=1}^{N} V\left(q_{j}-q_{j+1}\right), \quad V=\frac{J^{2}}{2} q^{2},
$$

после необходимой корректировки коэффициента $M$ при $q_{j}^{2}$ и предполагая граничные условия Дирихле $q_{0}=q_{N+1}=0$. Рассмотрим временную эволюцию Ланжевена, задаваемую стохастическими дифференциальными уравнениями

$$
d q_{j}=p_{j} d t, \quad d p_{j}=-\frac{\partial H}{\partial q_{j}} d t-\xi p_{j} d t+\gamma_{j}^{1 / 2} d B_{j}, \quad j=1,2, \ldots, N,
$$

где $B_{j}$ - независимые винеровские процессы (т.е. $\eta_{j}=d B_{j} / d t$ - независимые белые шумы), $\xi$ - взаимодействие между узлом $j$ и его термостатом, $\gamma_{j}=2 \xi T_{j}$, где $T_{j}-$ температура $j$-го резервуара. Как обычно, определим энергию одного осциллятора следующим образом:

$$
H_{j}(q, p)=\frac{1}{2} p_{j}^{2}+q_{j}^{2}+U\left(q_{j}\right)+\frac{1}{2} \sum_{l \neq j} V\left(q_{j}-q_{l}\right),
$$


где выражения для $U$ и $V$ следуют из (1) и из равенства $\sum_{j} H_{j}=H$. Тогда для энергетического тока получаем

$$
\left\langle\frac{d H_{j}}{d t}\right\rangle=\left\langle R_{j}\right\rangle-\left\langle\mathcal{F}_{\left.j \leftarrow-\mathcal{F}_{j \rightarrow}\right\rangle},\right.
$$

где $\langle\cdot\rangle$ - ожидание по шумовому распределению, $R_{j}$ - поток энергии между $j$-м резервуаром и $j$-м узлом,

$$
\left\langle R_{j}\right\rangle=\xi\left(T_{j}-\left\langle p_{j}^{2}\right\rangle\right),
$$

а энергетический ток в цепочке выражается следующим образом:

$$
\begin{aligned}
& \mathcal{F}_{j \rightarrow}=\sum_{l>j} \nabla V\left(q_{j}-q_{l}\right) \frac{p_{j}+p_{l}}{2}, \\
& \mathcal{F}_{j \leftarrow}=\sum_{l<j} \nabla V\left(q_{l}-q_{j}\right) \frac{p_{l}+p_{j}}{2} .
\end{aligned}
$$

Как хорошо известно, стационарное состояние характеризуется условием $\left\langle d H_{i} / d t\right\rangle=0$. По физическим причинам интересно рассмотреть "условие самосогласованости" вида $\left\langle R_{j}\right\rangle=0$ в стационарном состоянии. Для линейной динамики существование стационарного состояния и сходимость к нему при $t \rightarrow \infty$ являются давно решенными задачами (см., например, [9]).

\section{2. ИНТЕГРАЛЬНОЕ ПРЕДСТАВЛЕНИЕ}

Введем вектор $\phi=(q, p)$ в фазовом пространстве и запишем динамику в виде

$$
\dot{\phi}=-A \phi-\lambda \mathcal{P}^{\prime}(\phi)+\sigma \eta,
$$

где $A=A^{0}+\mathcal{J}, \sigma-(2 N \times 2 N)$-матрицы,

$$
A^{0}=\left(\begin{array}{cc}
0 & -I \\
\mathcal{M} & \Gamma
\end{array}\right), \quad \mathcal{J}=\left(\begin{array}{ll}
0 & 0 \\
J & 0
\end{array}\right), \quad \sigma=\left(\begin{array}{cc}
0 & 0 \\
0 & \sqrt{2 \Gamma \mathcal{T}}
\end{array}\right),
$$

$I$ - единичная $(N \times N)$-матрица, $J-(N \times N)$-матрица межчастичных взаимодействий, $\mathcal{M}, \Gamma$ и $\mathcal{T}$ - диагональные $(N \times N)$-матрицы: $M_{j l}=M \delta_{j l}, \Gamma_{j l}=\xi \delta_{j l}, \mathcal{T}=T_{j} \delta_{j l}$. Кроме того, $\eta$ - независимые белые шумы, $\mathcal{P}^{\prime}(\phi)-(2 N \times 1)$-матрица, для которой $\mathcal{P}^{\prime}(\phi)_{j}=0$ при $j=1,2, \ldots, N$ и

$$
\mathcal{P}^{\prime}(\phi)_{i}=\frac{d \mathcal{P}\left(\phi_{i-N}\right)}{d \phi_{i-N}}, \quad i=N+1, N+2, \ldots, 2 N .
$$

Далее мы будем использовать следующие индексные обозначения: $i$ для значений индекса во множестве $\{N+1, N+2, \ldots, 2 N\} ; j$ для значений во множестве $\{1,2, \ldots, N\}$ и $k$ для значений в $\{1,2, \ldots, 2 N\}$. Очевидные символы суммирования по повторяющимся индексам опускаются.

Во-первых, рассмотрим уравнение $(8)$ при $\mathcal{J} \equiv 0, \lambda=0$, т.е. систему с изолированными узлами (без взаимодействия между ними). Во-вторых, введем межчастичные взаимодействия и ангармонический потенциал с использованием теоремы Гирсанова, затем получим интегральное представление для корреляционных функций. 
Далее мы получим поток тепла для гармонической цепочки с помощью пертурбативного (но строгого) вычисления.

Справедлива следующая лемма.

ЛЕмма 1. Решение уравнения (8) при $\mathcal{J} \equiv 0$ является гауссовым процессом Орнштейна-Уленбека

$$
\phi(t)=e^{-t A^{0}} \phi(0)+\int_{0}^{t} d s e^{-(t-s) A^{0}} \sigma \eta(s),
$$

где при $\phi(0)=0$ двухточечная коррелячионная функция прощесса эволющионирует следующим образом:

$$
\begin{gathered}
\langle\phi(t) \phi(s)\rangle_{0} \equiv \mathcal{C}(t, s)= \begin{cases}e^{-(t-s) A^{0} \mathcal{C}(s, s),} & t \geqslant s, \\
\mathcal{C}(t, t) e^{-(s-t) A^{0^{\mathrm{T}}}}, & t \leqslant s,\end{cases} \\
\mathcal{C}(t, t)=\int_{0}^{t} d s e^{-s A^{0}} \sigma^{2} e^{-s A^{0^{\mathrm{T}}}} .
\end{gathered}
$$

Доказательство представляет собой простое упражнение по стохастическим дифференциальным уравнениям (см., например, [10], с. 74).

В простейшем случае, когда $\mathcal{J} \equiv 0$, при $t \rightarrow \infty$ имеем сходимость к гауссову распределению с двухточечной корреляционной функцией

$$
C=\int_{0}^{\infty} d s e^{-s A^{0}} \sigma^{2} e^{-s\left(A^{0}\right)^{\mathrm{T}}}=\left(\begin{array}{cc}
\frac{\mathcal{T}}{\mathcal{M}} & 0 \\
0 & \mathcal{T}
\end{array}\right)
$$

(см., например, [9]).

Решение уравнения (8) с межчастичным взаимодействием и ангармоническим потенциалом выводится из частного случая при $\mathcal{J} \equiv 0, \lambda=0$ с использованием теоремы Гирсанова, которая определяет меру $\rho$ для полного процесса в терминах меры $\mu_{\mathcal{C}}$, полученной при $\mathcal{J} \equiv 0, \lambda=0$. А именно, для $k$-точечной корреляционной функции имеем следующий результат.

Теорема 1. Для корреляционных функций кристаллической цепочки с резервуарами в каждом узле (1)-(3) имеется интегральное представление (в духе Фейнмана-Каца) вида

$$
\left\langle\varphi_{u_{1}}\left(t_{1}\right) \ldots \varphi_{u_{k}}\left(t_{k}\right)\right\rangle=\int \phi_{u_{1}}\left(t_{1}\right) \ldots \phi_{u_{k}}\left(t_{k}\right) e^{-W(\phi)} d \mu_{\mathcal{C}}, \quad t_{1}, \ldots, t_{k} \leqslant t
$$

¿əe

$$
\begin{gathered}
e^{-W(\phi)}=\exp \left[-F_{1}(\phi(t))+F_{1}(\phi(0))-\lambda F_{2}(\phi(t))+\lambda F_{2}(\phi(0))\right] \times \\
\quad \times \exp \left[-\int_{0}^{t} W_{J}(\phi(s)) d s-\int_{0}^{t} \lambda W_{\lambda}(\phi(s)) d s-\int_{0}^{t} \lambda W_{\lambda J}(\phi(s)) d s\right] \\
F_{1}(\phi(t))=\gamma_{i}^{-1} \phi_{i}(t) \mathcal{J}_{i j} \phi_{j}(t), \quad F_{2}(\phi(t))=\gamma_{i}^{-1} \mathcal{P}^{\prime}(\phi)_{i}(t) \phi_{i}(t) \\
W_{J}(\phi(s))=\gamma_{i}^{-1} \phi_{i}(s) \mathcal{J}_{i j} A_{j k}^{0} \phi_{k}(s)+\phi_{k}(s) A_{k i}^{0^{\mathrm{T}}} \gamma_{i}^{-1} \mathcal{J}_{i j} \phi_{j}(s)+ \\
+\frac{1}{2} \phi_{j^{\prime}}(s) \mathcal{J}_{j^{\prime} i}^{\mathrm{T}} \gamma_{i}^{-1} \mathcal{J}_{i j} \phi_{j}(s),
\end{gathered}
$$




$$
\begin{aligned}
\lambda W_{\lambda}(\phi(s))=\lambda & \gamma_{i}^{-1} \phi_{i}(s) \mathcal{P}^{\prime \prime}(\phi)_{i}(s) A_{i-N, k}^{0} \phi_{k}(s)+\lambda \gamma_{i}^{-1} \mathcal{P}^{\prime}(\phi)_{i}(s) A_{i k}^{0} \phi_{k}(s)+ \\
& +\frac{1}{2} \lambda^{2} \gamma_{i}^{-1}\left(\mathcal{P}^{\prime}(\phi)_{i}\right)^{2}(s), \\
\lambda W_{\lambda J}(\phi(s))=\lambda & \gamma_{i}^{-1} \mathcal{P}^{\prime}(\phi)_{i}(s) \mathcal{J}_{i j} \phi_{j}(s),
\end{aligned}
$$

где $\phi$ - решение (определяемое леммой 1) для процесса (8) при $\mathcal{J} \equiv 0, \lambda=0, \varphi-$ решение для полного процесса (8); двухточечная корреляиионная функция $\mathcal{C}$ имеет вид (12), (13).

ДокАзАтЕЛьство. Используя теорему Гирсанова (формулу Камерона-Мартина), можно записать меру $\rho$ для нового процесса как "возмущение" меры $\mu_{\mathcal{C}}$, связанной с процессом при $J \equiv 0, \lambda=0^{1)}$. А именно, для любого измеримого множества $A$ имеем $\rho(A)=E_{0}\left(1_{A} Z(t)\right)$, где $E_{0}$ - ожидаемое значение для меры $\mu_{\mathcal{C}}$ (процесс (8) при $J \equiv 0, \lambda=0), 1_{A}$ обозначает характеристическую функцию, а

$$
\begin{gathered}
Z(t)=\exp \left[\int_{0}^{t} u \cdot d B-\frac{1}{2} \int_{0}^{t} u^{2} d s\right], \\
\gamma_{i}^{1 / 2} u_{i}=-\mathcal{J}_{i k} \phi_{k}-\lambda \mathcal{P}^{\prime}(\phi)_{i}
\end{gathered}
$$

(подынтегральные произведения здесь лежат в $\mathbb{R}^{2 N}$ ). Заметим, что $u_{i}$ не равно нулю только при $i>N$ (т.е. при $i \in\{N+1, N+2, \ldots, 2 N\})$. Учитывая формулу (17), получаем

$$
\begin{aligned}
u_{i} d B_{i} & =\gamma_{i}^{-1 / 2} u_{i} \cdot \gamma_{i}^{1 / 2} d B_{i}=\gamma_{i}^{-1 / 2} u_{i} \cdot\left(d \phi_{i}+A_{i k}^{0} \phi_{k} d t\right)= \\
& =\left(-\gamma_{i}^{-1} \mathcal{J}_{i j} \phi_{j}-\gamma_{i}^{-1} \lambda \mathcal{P}^{\prime}(\phi)_{i}\right)\left(d \phi_{i}+A_{i k}^{0} \phi_{k} d t\right) .
\end{aligned}
$$

Используя формулу Ито, имеем

$$
-\gamma_{i}^{-1} \mathcal{J}_{i j} \phi_{j} d \phi_{i}=-d F_{1}-\gamma_{i}^{-1} \phi_{i} \mathcal{J}_{i j} A_{j k}^{0} \phi_{k} d t, \quad F_{1}(\phi)=\gamma_{i}^{-1} \phi_{i} \mathcal{J}_{i j} \phi_{j}
$$

Аналогично получаем выражение для $W(\phi)$.

Обоснуем теперь использование теоремы Гирсанова в случае некоторой жесткой ангармоничности.

ЛЕмма 2. Интегралъное представление для корреляционных функиий, задаваемое приведенными выше выражениями, имеет место и в случае жестко ангармонического потенциала $\mathcal{P}(\phi)=\phi^{4}$.

ДокАзАТЕльство. Для доказательства воспользуемся теоремой 8.6.8 в [10]. Сначала заметим, что $\varphi$ и $\phi$ (в том виде, как они были определены) являются соответственно процессом Ито и диффузией Ито. Тогда для применения теоремы Гирсанова надо показать, что $Z(t)$ есть мартингал по отношению к $\sigma$-алгебре, которую порождают $\phi(t)$ и $\mu_{\mathcal{C}}$. Будем действовать следующим образом. Определим процесс Ито

$$
d X(t)=-\frac{1}{2} u^{2} d t+u \cdot d B, \quad X(0)=0
$$

\footnotetext{
1) Использование этой теоремы вполне стандартно в случае, например, ограниченного ангармонического потенциала. В других случаях требуется некоторое дополнительное обоснование (см. ниже лемму 2).
} 
где функция $u$ введена в (17). Тогда $Z(t)=e^{X(t)}$ также является процессом Ито (см., например, теорему 4.1.2 в [10]), для которого имеем

$$
d Z(t)=Z(t) u \cdot d B(t) \Rightarrow Z(t)=1+\int_{0}^{t} Z(s) u(s) \cdot d B(s) .
$$

Из этого выражения следует, что $Z(t)$ - мартингал для $Z u(\phi)$, неупреждающий и квадратично-интегрируемый (см., например, следствие 3.2.6 в [10]). Поскольку процесс $\phi$ допускает континуальную реализацию, заключаем, что $Z(t)$ ограничен (заметим, что $u$ квадратично-интегрируемо), а потому $u Z$ квадратично-интегрируемо, т.e.

$$
E_{0}\left(\int_{0}^{t} u^{2}(s) Z^{2}(s) d s\right)<\infty
$$

\section{3. ТЕПЛОПРОВОДНОСТЬ ДЛЯ ГАРМОНИЧЕСКОЙ ЦЕПОЧКИ}

Обратимся теперь к гармонической цепочке и исследуем тепловой поток в стационарном состоянии. Сформулируем наш основной результат.

Теорема 2. Для гармонической цепочки осиилляторов с резервуарами в каждом узле (1)-(3) в случае взаимодействия соседних осцилляторов, т.е. при $J_{j l}=$ $J\left(\delta_{l, j+1}+\delta_{l, j-1}\right)$, где $J<J_{0}$ для некоторого малого $J_{0}$, выполнен закон Фуръе

$$
\mathcal{F}=\lim _{t \rightarrow \infty}\left\langle\mathcal{F}_{j \rightarrow}\right\rangle=-\frac{\chi}{N-1}\left(T_{N}-T_{1}\right)
$$

с условием “самосогласованности" $\lim _{t \rightarrow \infty}\left\langle R_{j}\right\rangle=0$ для внутренних узлов $j$, причем теплопроводность х задается следующим образом:

$$
\chi=\frac{J^{2}}{2 \xi M}+\mathcal{O}\left(J^{3}\right) .
$$

ЗАмечАниЕ 1. Теорема, устанавливающая конечность теплопроводности для рассмотренной модели, была, как мы уже отмечали, сформулирована ранее в [4], причем с не обязательно малым межчастичным потенциалом для соседних узлов. Наша цель, как мы уже подчеркивали, состоит не в том, чтобы дать второе доказательство, а в том, чтобы представить более общий подход, распространяемый на ангармонические цепочки.

ЗАмечАниЕ 2. Согласно описанным ниже шагам можно также исследовать тепловой поток для слабых межчастичных взаимодействий за пределами соседних узлов, например, при $\sup _{l} \sum_{j} J_{l j} \leqslant J_{0}$.

Чтобы исследовать тепловой поток в стационарном состоянии, связанном с $\lim _{t \rightarrow \infty}\left\langle\varphi_{u}(t) \varphi_{v}(t)\right\rangle$, и доказать сформулированную теорему, поступим следующим образом. Прежде всего заметим, что

$$
e^{-t A^{0}}=e^{-t \xi / 2} \operatorname{ch}(t \rho)\left\{\left(\begin{array}{ll}
I & 0 \\
0 & I
\end{array}\right)+\frac{\operatorname{th}(t \rho)}{\rho}\left(\begin{array}{cc}
\frac{\xi}{2} & I \\
-\mathcal{M} & -\frac{\xi}{2}
\end{array}\right)\right\},
$$

где $\rho=\left((\xi / 2)^{2}-M\right)^{1 / 2}$. Это можно показать, например, диагонализацией $A^{0}$ (см. также приложение в [4]). Заметим, кроме того, что

$$
\mathcal{C}(t, s)=\exp \left[-(t-s) A^{0}\right] C+\mathcal{O}(\exp [-(t+s) \xi / 2]),
$$


где второй член в формуле для корреляционной функции обращается в нуль в пределе при $t \rightarrow \infty, C$ является корреляционной функцией (14).

Для вычисления двухточечной корреляционной функции, а тем самым и потока тепла (см. теорему 1 в случае $k=2$ и $\lambda=0$ ), разложим член $e^{-W}$ в степенной ряд $e^{-W}=\sum_{n=0}^{\infty}(-W)^{n} / n$ ! и вычислим связные фейнмановские графы для $\left\langle\varphi_{u}(t) \varphi_{v}(t)\right\rangle$, используя теорему Вика (из-за нормировочного множителя остаются только связные графы). Поскольку в экспоненте имеются квадратичные по $\phi$ члены (см. приведенные выше формулы для $F$ и $W$ ), имеем

$$
\left|\int \frac{\phi^{2 n}}{n !} d \mu_{\mathcal{C}}\right|=|\frac{1}{n !} \sum_{k=1}^{(2 n-1) ! !} \underbrace{(\mathcal{C C} \ldots \mathcal{C})}_{n}| \leqslant \frac{(2 n) ! !}{n !}|\mathcal{C} \ldots \mathcal{C}|=2^{n}|\mathcal{C} \ldots \mathcal{C}| .
$$

Тем самым, для контроля за разложением требуется только оценка для $n$ сверток двухточечных корреляционных функций $\mathcal{C}$ типа $c^{n} J^{n}$, где $c$ - некоторая постоянная. Таким образом, по крайней мере для малых $J$ этот простой анализ приводит к сходимости ряда теории возмущений. Для получения нужной оценки используем формулы $(20),(21)$ для $\mathcal{C}$, приведенную ниже лемму 3 и следующие неравенства:

$$
\|\mathcal{C}(t, t)\| \leqslant c_{1}\left(1-e^{-2 \alpha t}\right), \quad\left\|e^{-t A^{0}}\right\| \leqslant c_{2} e^{-\alpha t}, \quad\left\|A^{0}\right\| \leqslant c_{3},
$$

где $\alpha=\min \{\xi / 2, M / \xi\}\left(\|\cdot\|\right.$ - норма в пространстве $\left.M_{2 N \times 2 N}(\mathbb{R})\right)$.

Лемма 3. Пусть $I_{t}$ имеет вид

$$
I_{t}=\int_{0}^{t} e^{-\alpha\left|t-s_{1}\right|} e^{-\alpha\left|s_{1}-s_{2}\right|} \ldots e^{-\alpha\left|t-s_{n}\right|} d s_{1} \ldots d s_{n}, \quad \alpha>0 .
$$

Тогда $\lim _{t \rightarrow \infty} I_{t} \leqslant\left(c_{\alpha}\right)^{n}$, где $c_{\alpha}$ не зависит от $n$.

ДокАЗАТЕЛЬСтво. При $f(x)=e^{-\alpha|x|}$ имеем

$$
\tilde{f}(p) \equiv \frac{1}{\sqrt{2 \pi}} \int_{-\infty}^{\infty} e^{-i p x} e^{-\alpha|x|} d x=\frac{1}{\sqrt{2 \pi}} \frac{2 \alpha}{\alpha^{2}+p^{2}} .
$$

Кроме того,

$$
\begin{aligned}
I_{t} & \leqslant \frac{1}{2} \int_{-\infty}^{\infty} e^{-\alpha\left|t-s_{1}\right|} e^{-\alpha\left|s_{1}-s_{2}\right|} \ldots e^{-\alpha\left|t-s_{n}\right|} d s_{1} \ldots d s_{n}= \\
& =\frac{1}{2} \int_{-\infty}^{\infty} f\left(t-s_{1}\right) f\left(s_{1}-s_{2}\right) \ldots f\left(s_{n}-t\right) d s_{1} \ldots d s_{n}= \\
& =\frac{1}{2} \underbrace{f * f * \cdots * f}_{n+1}(0)=\frac{1}{2}(2 \pi)^{(n-1) / 2} \int_{-\infty}^{\infty}(\tilde{f}(p))^{n+1} d p,
\end{aligned}
$$

где $*$ обозначает свертку и в последнем равенстве использована теорема Парсеваля. Поэтому

$$
\lim _{t \rightarrow \infty} I_{t} \leqslant \frac{1}{2}(2 \pi)^{(n-1) / 2} \int_{-\infty}^{\infty} \frac{1}{(2 \pi)^{(n+1) / 2}}\left(\frac{2 \alpha}{\alpha^{2}+p^{2}}\right)^{n+1} d p \leqslant\left(c_{\alpha}\right)^{n} .
$$


Таким образом, используя формулы (20), (21) для двухточечной корреляционной функции и лемму 3 , можно оценить члены порядка $J^{2}$ и выше в представлении для двухточечной функции следующим образом:

$$
\sum_{n=2}^{\infty}\left(c^{\prime} J\right)^{n} \leqslant c^{\prime \prime} J^{2}
$$

где $c^{\prime}$ и $c^{\prime \prime}-$ некоторые величины, не зависящие от $n$.

Для доказательства теоремы 2 вычислим двухточечную корреляционную функцию с точностью до первого порядка по $J$. Выполняя простые вычисления для $\left\langle\varphi_{u} \varphi_{v}\right\rangle \equiv \lim _{t \rightarrow \infty}\left\langle\varphi_{u}(t) \varphi_{v}(t)\right\rangle$, получаем

$$
\left\langle\varphi_{u} \varphi_{v}\right\rangle=\left\{\begin{array}{rlrl}
\frac{1}{2 \xi M}\left[\mathcal{J}_{v+N, u-N} T_{u-N}-\mathcal{J}_{u, v} T_{v}\right] & \text { при } \quad u & \in\{N+1, N+2, \ldots, 2 N\}, \\
& v & \in\{1,2, \ldots, N\}, \\
T_{u-N} \delta_{u, v} & \text { при } & u, v & \in\{N+1, N+2, \ldots, 2 N\} .
\end{array}\right.
$$

Поэтому из (7) имеем

$$
\mathcal{F}_{j \rightarrow}=\sum_{r>j} \mathcal{J}_{j+N, r}\left(\varphi_{j}-\varphi_{r}\right) \frac{\left(\varphi_{j+N}+\varphi_{r+N}\right)}{2}, \quad r \in\{1,2, \ldots, N\},
$$

и (для $\left\langle\varphi_{u} \varphi_{v}\right\rangle$ с точностью до первого порядка по $\left.J\right)$

$$
\left\langle\mathcal{F}_{j \rightarrow}\right\rangle=\sum_{r>j} \frac{\left(\mathcal{J}_{j+N, r}\right)^{2}}{2 \xi M}\left(T_{r}-T_{j}\right) .
$$

Тем самым, для взаимодействий в соседних узлах

$$
\mathcal{F}_{j \rightarrow j+1}=\left\langle\mathcal{F}_{j \rightarrow}\right\rangle=\frac{\left(\mathcal{J}_{j+N, j+1}\right)^{2}}{2 \xi M}\left(T_{j+1}-T_{j}\right) .
$$

Вычисление в первом порядке теории возмущений (28) по-прежнему дает $\lim _{t \rightarrow \infty}\left\langle R_{j}(t)\right\rangle=0$.

Таким образом, условие стационарности $\left\langle d H_{i} / d t\right\rangle=0$ приводит к равенствам

$$
\mathcal{F}_{1 \rightarrow 2}=\mathcal{F}_{2 \rightarrow 3}=\cdots=\mathcal{F}_{N-1 \rightarrow N} \equiv \mathcal{F} .
$$

Поэтому в более простом случае одинаковых взаимодействий между любыми двумя соседними узлами, т.е. при $J=\mathcal{J}_{j+N, j+1}$ для всех $j$, получаем (для $\left\langle\varphi_{u} \varphi_{v}\right\rangle$ с точностью до первого порядка по $J$ )

$$
\mathcal{F}_{\rightarrow}=\chi \frac{T_{N}-T_{1}}{N-1}, \quad \chi=\frac{J^{2}}{2 \xi M} .
$$

Из (27) и (29) легко видеть, что вычисление оставшихся членов (высших порядков по $J$ ) даст вклад $J \times c J^{2}=c J^{3}$ в $\chi$ (температурные члены $T_{j+1}-T_{j}$ можно найти с помощью пертурбативного анализа, вычисляя произведения величин $C$, двухточечной корреляционной функции $\mathcal{C}(13)$, появляющейся в гауссовом взаимодействии, и членов $\gamma_{i}$, возникающих из разложения для $\left.Z(t)\right)$. Таким образом, теорема 2 доказана. 
ЗАмечАниЕ 3. В случае ангармонических членов в формулах для $W$ и $F$ стандартное разложение не приводит к нужному результату, но мы надеемся развить пертурбативный подход с использованием некоторого кластерного разложения, которое хорошо известно в теории поля и статистической механике. В более простом случае неконсервативной нелинейной стохастической динамической модели, описывающей систему в контакте с тепловыми резервуарами при той же температуре, а потому стремящейся к равновесию, сходящееся кластерное разложение приведено в [11], а убывание четырехточечной функции исследовано в [12], [13]. Полный и строгий результат в [12] добавляет лишь малые поправки к пертурбативному вычислению первого порядка [13].

Благодарности. Работа выполнена при частичной поддержке CNPq и CAPES (Бразилия).

\section{Список литературы}

[1] F. Bonetto, J. L. Lebowitz, L. Rey-Bellet, "Fourier's law: a challenge to theorists", Mathematical Physics, eds. A. Fokas, A. Grigoryan, T. Kibble, B. Zegarlinski, Imp. Coll. Press, London, 2000, 128-150.

[2] S. Lepri, R. Livi, A. Politi, Phys. Rep., 377:1 (2003), 1-80.

[3] Z. Rieder, J. L. Lebowitz, E. Lieb, J. Math. Phys., 8:5 (1967), 1073-1078.

[4] F. Bonetto, J. L. Lebowitz, J. Lukkarinen, J. Stat. Phys., 116:1-4 (2004), 783-813.

[5] E. Pereira, R. Falcao, Phys. Rev. E, 70:4 (2004), 046105.

[6] E. Pereira, R. Falcao, Phys. Rev. Lett., 96:10 (2006), 100601.

[7] A. Francisco Neto, H. C. F. Lemos, E. Pereira, J. Phys. A, 39:30 (2006), 9399-9410.

[8] F. Barros, H. C. F. Lemos, E. Pereira, Phys. Rev. E, 74:5 (2006), 052102.

[9] J. Snyders, M. Zakai, SIAM J. Appl. Math., 18:3 (1970), 704-714.

[10] B. Øksendal, Stochastic Differential Equations: An Introduction with Applications, Springer, Berlin, 2003.

[11] J. Dimock, J. Stat. Phys., 58:5-6 (1990), 1181-1207.

[12] P. A. Faria da Veiga, M. O'Carroll, E. Pereira, R. Schor, Comm. Math. Phys., 220:2 (2001), 377-402.

[13] R. Schor, J. C. A. Barata, P. A. Faria da Veiga, E. Pereira, Phys. Rev. E, 59:3 (1999), 2689-2694. 